\title{
Association of total body and visceral fat mass with iron deficiency in preadolescents: the Healthy Growth Study
}

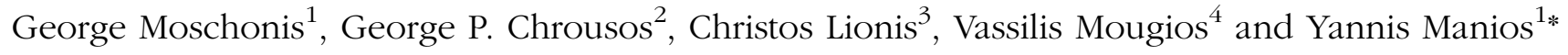 \\ on behalf of the Healthy Growth Study group \\ ${ }^{1}$ Department of Nutrition and Dietetics, Harokopio University of Athens, 70, El. Venizelou Avenue, 17671 Kallithea-Athenes, \\ Greece \\ ${ }^{2}$ First Department of Pediatrics, Athens University Medical School, Aghia Sophia Children's Hospital, Athens, Greece \\ ${ }^{3}$ Clinic of Social and Family Medicine, School of Medicine, Heraklion, University of Crete, Crete, Greece \\ ${ }^{4}$ Department of Physical Education and Sport Science, Aristotle University of Thessaloniki, Thessaloniki, Greece
}

(Submitted 27 April 2011 - Final revision received 4 October 2011 - Accepted 5 October 2011 - First published online 16 November 2011)

\begin{abstract}
The aim of the present study was to examine the associations of obesity, percentage body fat and visceral fat mass with body Fe status in a representative sample of 1493 schoolchildren aged 9-13 years. Anthropometric, body composition, biochemical, clinical (Tanner stage, age of menarche) and dietary intake data were collected. Fe deficiency (ID) was defined as transferrin saturation (TS) $<16 \%$; and Fe-deficiency anaemia (IDA) as ID with $\mathrm{Hb}<120 \mathrm{~g} / \mathrm{l}$. Obese boys and girls and those in the highest quartiles of percentage body fat mass had significantly higher levels of serum ferritin $(P \leq 0.05)$ compared to their normal-weight peers and those in the corresponding lowest quartiles. Similarly, obese boys and girls and those in the highest quartiles of percentage body fat and visceral fat mass had significantly lower levels of TS $(P \leq 0.05)$ compared to normal-weight children and those in the corresponding lowest quartiles. The prevalence of ID and IDA was significantly higher in boys and girls in the highest quartiles of percentage body fat than in peers in the lowest quartile. Higher quartiles of percentage body fat and visceral fat mass were the main significant predictors of ID in boys, after controlling for other important confounders, with OR of $2 \cdot 48$ (95\% CI, 1.26, 4.88) and 2.12 (95\% CI, 1.07, 4.19), respectively. Similar significant associations were observed for girls. In conclusion, percentage body fat and visceral fat mass were positively associated with ID in both sexes of preadolescents. These associations might be attributed to the chronic inflammation induced by excess adiposity.
\end{abstract}

Key words: Iron deficiency: Obesity: Children: Adipose tissue

Many reports have indicated that the prevalence of obesity in childhood and adolescence has been increasing worldwide at an alarming rate ${ }^{(1,2)}$. On the basis of recent studies, the number of overweight children has doubled and the number of overweight adolescents has tripled since $1970^{(3)}$. Following the worldwide trends, obesity among Greek children and adolescents is also on the rise over the last 30 years ${ }^{(4)}$. It is well established that excess adiposity in both adults and children is strongly associated with several metabolic complications (i.e. metabolic syndrome, insulin resistance, dyslipidaemias) ${ }^{(5,6)}$. In addition, although contradictory at first sight, obesity has also been associated with nutritional deficiencies $^{(7,8)}$. Specifically, overweight and obese individuals seem to be at higher risk of Fe deficiency (ID) than those having normal body weight ${ }^{(9-11)}$. These findings were consistent in both children ${ }^{(12-15)}$ and adults ${ }^{(15-17)}$.
Prevention of ID is crucial from a public health perspective, because it is associated with behavioural and cognitive delays in infancy and early childhood, such as impaired learning ${ }^{(18)}$, decreased school achievements ${ }^{(19,20)}$ and lower scores on tests of mental and motor development ${ }^{(21)}$. The aetiology of this phenomenon remains uncertain. Suggested contributing factors are poor Fe intake, repeated short-term restrictive diets, increases in blood volume when children enter adolescence, early onset of menstruation, limited physical activity, rapid growth and genetics ${ }^{(12,14)}$. However, these factors seem not to be significant predictors of the low serum Fe levels observed in overweight and obese individuals ${ }^{(16)}$. In contrast, ID could partially be explained by the fact that obesity is a low-grade chronic inflammatory state ${ }^{(22)}$. In particular, studies show that increased levels of inflammatory biomarkers, such as C-reactive protein, are inversely associated with serum Fe

Abbreviations: ID, Fe-deficiency; IDA, Fe-deficiency anaemia; TIBC, total Fe-binding capacity; TS, transferrin saturation; WC, waist circumference. 
levels in centrally obese adolescents ${ }^{(10)}$. The hypothesis that the association between ID and obesity is mediated by an obesity-induced low-grade chronic inflammation is strengthened by the higher levels of serum ferritin usually observed in overweight and obese individuals. Although ferritin serves as an index of Fe stores in the body, it is also an acutephase protein that increases in inflammatory states, such as excess visceral fat accumulation ${ }^{(23)}$.

The studies showing an inverse association between adiposity and Fe status in children and adolescents ${ }^{(10,12,14)}$ have relied on BMI. However, BMI is not always a direct measure of adiposity, especially in children ${ }^{(24)}$. Furthermore, to our knowledge based on the available literature, no study so far has ever examined the relationship between more direct measures of adiposity and Fe status. Such studies may explore a more accurate association between obesity and ID in children and highlight further the discussion of dietary recommendations of $\mathrm{Fe}$ intake in them. The present study reports on the associations between central obesity, percentage body fat and visceral fat mass and ID in a representative sample of preadolescent Greek children.

\section{Methods and procedures}

\section{Sampling}

The Healthy Growth Study was a large-scale, cross-sectional, epidemiological study initiated in May 2007. This study was conducted according to the guidelines laid down in the Declaration of Helsinki and all procedures involving human subjects were approved by the Ethics Committee of Harokopio University of Athens. Approval to conduct the study was also granted by the Greek Ministry of National Education. The population under study comprised schoolchildren aged 9-13 years, attending the 5th and 6th grades of primary schools located in municipalities within the prefectures of Attica, Etoloakarnania, Thessaloniki and Iraklio. The sampling of schools was random, multi-stage and stratified by parents' educational level and total population of students attending schools within these municipalities. Specifically, the municipalities in the prefectures under study were divided into three groups on the basis of average educational level of their adult population (25-65 years old) that was estimated from data provided by the National Statistical Service of Greece (2001 census). This procedure yielded two parents' education cut-off points that allowed us to categorise municipalities into three different socio-economic levels, i.e. higher, middle and lower. Consequently, again on the basis of data from the National Statistical Service, a certain number of municipalities, proportional to the size of their preadolescent population (9-13 years old), was randomly selected from each socio-economic level. Finally, a number of schools were randomly selected from each municipality, proportional to the population of schoolchildren registered in the 5 th and 6th grades, according to data obtained from the Greek Ministry of Education.

All seventy-seven primary schools that were invited to participate in the study responded positively. Weight and height were measured in all pupils attending the 5th and 6th grades in these primary schools as part of a school-based health and nutrition education programme. Full medical examination (i.e. anthropometric and body composition measurements, blood collection, clinical examination etc.) and questionnaire data were obtained from a subgroup of pupils whose parents signed an informed consent form. Signed parental consent forms were collected for 2655 out of 4145 children (response rate $64 \cdot 1 \%$ ). Still no significant differences with respect to parental educational level were observed between families that consented to data collection and those that did not.

\section{Physical examination and anthropometry}

Participants underwent a physical examination by two trained members of the research team. The protocol and equipment used were the same in all schools. Weight was measured to the nearest $10 \mathrm{~g}$ using a Seca digital scale (Seca Alpha, Model 770, Hamburg, Germany). Pupils were weighed without shoes in the minimum clothing possible. Height was measured to the nearest $0 \cdot 1 \mathrm{~cm}$ using a commercial stadiometer (Leicester Height Measure; Invicta Plastics, Oadby, UK), with the pupil standing barefoot, keeping shoulders in a relaxed position, arms hanging freely and head in Frankfurt horizontal plane. Weight and height were used to calculate BMI using Quetelet's equation (weight $(\mathrm{kg}) /$ height $(\mathrm{m})^{2}$ ). The International Obesity Task Force cut-off points ${ }^{(25,26)}$ were used to categorise participants as 'normal weight', 'overweight' or 'obese'. Waist circumference (WC) was measured to the nearest $0 \cdot 1 \mathrm{~cm}$ with the use of a non-elastic tape (Hoechstmass, Germany), with the pupil standing, at the end of a gentle expiration after placing the measuring tape on a horizontal plane around the trunk, at the level of umbilicus midway, between the lower rib margin and the iliac crest. The age- and sex-specific WC percentiles were used for the classification of central obesity ( $\geq 90$ th percentile) ${ }^{(27)}$. One well-trained and experienced female paediatrician in each prefecture determined pubertal maturation (Tanner stage) after thorough visual inspection of breast development in girls and genital development in boys $^{(28)}$. Finally, each girl was asked by the paediatrician about her menstruation status and age of menarche.

\section{Assessment of percentage body fat and visceral fat mass}

Bioelectrical impedance analysis was used for the assessment of percentage body fat (Akkern BIA 101; Akkern Srl., Florence, Italy) and for abdominal-visceral fat mass (Tanita Viscan AB-140, Kowloon, Hong Kong). In abdominal bioelectrical impedance analysis, an electric current is passed between the regions near the umbilicus and spinal cord at the umbilicus level, and the voltage generated in the lateral abdomen is recorded. Because the equipotential line that passes through visceral fat appears on the lateral abdominal surface, the amount of visceral fat can be estimated by measurement of the voltage generated at this location using a regression equation determined by computed tomography ${ }^{(29)}$. Participants were instructed to abstain from any food or liquid 
intake and from any intensive exercise for $4 \mathrm{~h}$ before measurement. They were also instructed not to wear any metallic object during measurement. The assessments took place with the pupils lying on a non-conductive surface at ambient room temperature. Percentage body fat was calculated from the resistance and reactance values using valid equations derived from a similar preadolescent population ${ }^{(30)}$, while visceral fat mass was read directly from the instrument in a rating scale from 1 to 59 units, with 0.5 increments. On the basis of these data, children were categorised into four sex-specific quartiles of percentage body fat and visceral fat mass. As there were only two Tanita Viscan devices available, data on trunk-visceral fat mass were collected for a representative sub-sample of 1500 children.

\section{Biochemical indices}

Blood samples were obtained for biochemical and haematological screening tests between 08.30 and 10.30 hours after a $12 \mathrm{~h}$ overnight fast. Reminders were distributed the previous day to both parents and children in order to ensure compliance with fasting. Professional staff performed venepuncture, using two types of test tubes, one of which contained EDTA, to obtain a maximum of $10 \mathrm{ml}$ blood. The EDTA-blood was transferred on the same day of collection to a local laboratory, where it was analysed in a CELL-DYN haematological autoanalyser (Abbott Diagnostics, Abbott Park, IL, USA) for the determination of haematological indices, including erythrocyte count, $\mathrm{Hb}$ and mean corpuscular volume. The remaining blood was collected in plain test tubes for the preparation of serum, which was divided into aliquots and stored at $-80^{\circ} \mathrm{C}$. When blood collection was completed in Aitoloakarnania, Thessaloniki and Iraklio, all serum samples were transported in dry ice to the Laboratory of Nutrition and Clinical Dietetics at Harokopio University, where biochemical analyses and central storage of back-up samples at $-80^{\circ} \mathrm{C}$ took place. Serum Fe and total Fe-binding capacity (TIBC) levels were determined by colorimetric assays (Roche Diagnostics SA, Basel, Switzerland). Transferin saturation (TS) was calculated by dividing serum Fe by TIBC and multiplying by 100. Finally, serum ferritin was measured by using a chemiluminescence immunoassay (Siemens Healthcare Diagnostics, Tarrytown, NY, USA).

Fe deficiency (with or without anaemia) and Fe-deficiency anaemia (IDA) were defined using the following age- and sex-specific thresholds proposed by UNICEF and the $\mathrm{WHO}^{(31)}$ : ID was defined as TS $<16 \%$; IDA was defined as TS $<16 \%$ and $\mathrm{Hb}$ concentration $<120 \mathrm{~g} / \mathrm{l}$, which is the threshold value for anaemia for children aged 9-13 years. The Mentzer Index (MCV (fl)/RBC $(\mathrm{M} / \mu \mathrm{l}))^{(32)}$ was also calculated for all pupils participating in the present study to differentiate beta-thalassaemia from ID. On the basis of this index, children with thalassaemia minor (eighteen cases) were excluded from further analysis.

\section{Dietary assessment}

Dietary intake data were obtained by trained dieticians and nutritionists via morning interviews with the children at school-site for two consecutive weekdays and one weekend day, using the $24 \mathrm{~h}$ recall technique. Specifically, all study participants were asked to describe the type and amount of foods, as well as all beverages consumed during the previous day, provided that it was a usual day according to the participant's perception. To improve the accuracy of food description, standard household measures (cups, tablespoons, etc.) and food models were used to define amounts where appropriate. At the end of each interview, the interviewers, who were dieticians rigorously trained to minimise the interviewer's effect, reviewed the collected data with the respondent in order to clarify entries, servings and possible forgotten foods. Food intake data were analysed using the Nutritionist $\mathrm{V}$ diet analysis software (version 2.1, 1999; First Databank, San Bruno, CA, USA), which was extensively amended to include traditional Greek recipes, as described $^{(33)}$. Furthermore, the database was updated with nutritional information of processed foods provided by independent research institutes, food companies and fastfood chains.

\section{Socio-economic and demographic variables}

Family socio-economic and demographic data (i.e. total years of education for the father and mother and annual family income) were collected during scheduled interviews at the school with the parents (mainly with the mother). For those parents not able to attend (approximately $5 \%$ of the total sample), data were collected via telephonic interviews. All interviews were conducted with the use of a standardised questionnaire by a research team that was rigorously trained to minimise the interviewer's effect.

\section{Statistical analysis}

Statistical analysis was conducted for the sub-sample of children with full anthropometric, biochemical, dietary, body composition and socio-economic data and no thalassaemia minor ( $n$ 1493). Continuous variables were expressed as mean values and standard deviations and categorical variables were reported as frequencies (\%). Comparisons between levels of the continuous variables were conducted using Student's $t$ test or ANOVA, using the Bonferroni correction for post hoc multiple comparisons among groups. Comparisons between levels of the categorical variables were conducted using the $\chi^{2}$ test or the Fisher's exact test, as appropriate. The two-sample $Z$-test was also used to perform pair-wise comparisons of the prevalence of ID and IDA between quartiles of percentage body fat and visceral fat mass. In order to test the effect of the independent variables examined on ID, multivariate logistic regression analysis was conducted and adjusted OR with 95\% CI were computed. All reported $P$ values were based on twosided tests. The level of statistical significance was set at $\alpha=0 \cdot 05$. Statistical analysis was conducted using STATA (Stata Corporation, College Station, TX, USA) for the twosample $Z$-test and SPSS version 17.0 (SPSS, Inc., TX, USA) for all other tests. 


\section{Results}

The sample consisted of 1493 children attending the 5th and 6 th grades of primary school. Table 1 presents the main descriptive characteristics of the study population. The age of the study participants was $11 \cdot 2$ (SD 0.7 ) years. No significant differences were found between boys and girls with respect to nationality and socio-economic characteristics (i.e. socio-economic level of school region, paternal and maternal educational level and family income). Similarly, no significant sex differences were found with respect to the prevalence of ID. On the other hand, more girls than boys were found to be at Tanner stages $3-5(P<0.05)$, while the prevalence of obesity was higher in boys than in girls $(12 \cdot 5 v \cdot 10 \cdot 2 \%, P<0.05)$.

Table 1. Descriptive characteristics of the study population

\begin{tabular}{|c|c|c|c|}
\hline & $\begin{array}{c}\text { Percentage } \\
\text { of boys }(n 740)\end{array}$ & $\begin{array}{l}\text { Percentage } \\
\text { of girls }(n 753)\end{array}$ & $\begin{array}{c}\text { Total } \\
\text { percentage } \\
(n 1493)\end{array}$ \\
\hline \multicolumn{4}{|l|}{ Age (years) } \\
\hline $9-11$ & 38.7 & $39 \cdot 1$ & 38.9 \\
\hline $11-13$ & $61 \cdot 3$ & $60 \cdot 9$ & $61 \cdot 1$ \\
\hline \multicolumn{4}{|l|}{ SEL of school } \\
\hline Lower & $25 \cdot 9$ & $24 \cdot 8$ & $25 \cdot 4$ \\
\hline Medium & $28 \cdot 5$ & $30 \cdot 8$ & $29 \cdot 7$ \\
\hline Higher & 45.5 & 44.4 & 44.9 \\
\hline \multicolumn{4}{|l|}{ Grade } \\
\hline 5th & $47 \cdot 0$ & 47.4 & $47 \cdot 2$ \\
\hline 6th & $53 \cdot 0$ & $52 \cdot 6$ & $52 \cdot 8$ \\
\hline \multicolumn{4}{|l|}{ Nationality } \\
\hline Greek & $89 \cdot 7$ & $87 \cdot 8$ & 88.7 \\
\hline Other & $10 \cdot 3$ & $12 \cdot 2$ & $11 \cdot 3$ \\
\hline \multicolumn{4}{|l|}{ Tanner stage } \\
\hline 1 & 44.8 & $21 \cdot 6^{\star}$ & $32 \cdot 8$ \\
\hline 2 & $43 \cdot 4$ & $37 \cdot 6^{*}$ & $40 \cdot 4$ \\
\hline 3 & $10 \cdot 6$ & $26 \cdot 6^{*}$ & $18 \cdot 8$ \\
\hline 4 & $1 \cdot 1$ & $10 \cdot 9^{\star}$ & $6 \cdot 2$ \\
\hline 5 & 0.1 & $3.3^{*}$ & 1.8 \\
\hline \multicolumn{4}{|l|}{ Menarche } \\
\hline Yes & - & $77 \cdot 4$ & - \\
\hline No & - & $22 \cdot 6$ & - \\
\hline \multicolumn{4}{|c|}{ Paternal education (years) } \\
\hline$<9$ & $25 \cdot 0$ & $27 \cdot 0$ & $26 \cdot 0$ \\
\hline $9-12$ & $40 \cdot 0$ & $37 \cdot 0$ & 38.5 \\
\hline$>12$ & 34.9 & $36 \cdot 0$ & 35.5 \\
\hline \multicolumn{4}{|c|}{ Maternal education (years) } \\
\hline$<9$ & $20 \cdot 7$ & $24 \cdot 1$ & $22 \cdot 4$ \\
\hline $9-12$ & 39.7 & 37.6 & 38.7 \\
\hline$>12$ & $39 \cdot 6$ & $38 \cdot 3$ & 38.9 \\
\hline \multicolumn{4}{|c|}{ Family income (€/year) } \\
\hline$<12000$ & $18 \cdot 3$ & 21.9 & $20 \cdot 1$ \\
\hline $12000-20000$ & $24 \cdot 8$ & 24.9 & 24.9 \\
\hline $20000-30000$ & $24 \cdot 1$ & $24 \cdot 2$ & $24 \cdot 2$ \\
\hline $30000-40000$ & $15 \cdot 9$ & $15 \cdot 6$ & $15 \cdot 7$ \\
\hline $40000-50000$ & 8.4 & $6 \cdot 2$ & $7 \cdot 3$ \\
\hline$>50000$ & $8 \cdot 4$ & $7 \cdot 2$ & $7 \cdot 8$ \\
\hline \multicolumn{4}{|l|}{ Weight groups } \\
\hline Normal-weight & $57 \cdot 0$ & $61 \cdot 3$ & $59 \cdot 2$ \\
\hline Overweight & $30 \cdot 5$ & $28 \cdot 6$ & 29.5 \\
\hline Obese & $12 \cdot 5$ & $10 \cdot 2^{*}$ & $11 \cdot 3$ \\
\hline \multicolumn{4}{|l|}{ ID } \\
\hline Normal & $85 \cdot 2$ & 85.4 & $85 \cdot 3$ \\
\hline ID (TS < 16\%) & $14 \cdot 8$ & $14 \cdot 6$ & $14 \cdot 7$ \\
\hline
\end{tabular}

SEL, socio-economic level; ID, Fe deficiency; TS, transferin saturation.

* Values were significantly different from boys $(P<0.05$, derived from the twosample Z-test for proportions).
Table 2 displays the biochemical indices of Fe status across weight groups in both boys and girls. In boys, serum Fe and TS differed significantly among weight groups $(P<0.05)$, being lower in obese and overweight than in normal-weight ones. In contrast, serum ferritin was lowest in normal-weight boys $(P<0.05)$. In girls, TS exhibited lower values in obese than in normal-weight girls. On the contrary, ferritin was higher in obese than in normal-weight girls; and TIBC exhibited higher values in obese and overweight than in normalweight girls. Moreover, the prevalence of ID was higher in obese boys and girls compared to their normal-weight peers ( $P=0.009$ in boys and $P=0.017$ in girls). Similarly, the prevalence of IDA was higher in obese than in overweight or normal-weight girls $(P=0 \cdot 002)$. Table 3 also presents the biochemical indices of $\mathrm{Fe}$ status in the categories of normal WC and central obesity. TS in centrally obese boys was significantly lower $(P=0.033)$ compared to peers of normal WC, while the prevalence of IDA was significantly higher in centrally obese boys than in boys of normal WC $(P=0.006)$. Furthermore, centrally obese girls exhibited higher prevalence of both ID and IDA than girls of normal WC $(P=0.001$ and 0.042, respectively).

Table 4 summarises the biochemical indices of $\mathrm{Fe}$ status across quartiles of percentage body fat. As far as boys were concerned, serum Fe and TS were found to differ significantly among quartiles, being lowest in the highest quartile. On the contrary, serum ferritin was lowest in the lowest quartile $(P=0 \cdot 001)$. Furthermore, the prevalence of ID and IDA was significantly higher for boys in the highest quartile of percentage body fat compared to the middle and lowest ones ( $P=0.005$ and 0.010 , respectively). In girls, serum Fe and TS exhibited lower values in the highest compared to the lowest quartile, whereas TIBC and ferritin exhibited higher values in the highest compared to the lowest quartile. Furthermore, the prevalence of ID and IDA was significantly higher for girls in the highest quartile of percentage body fat than for those in the lowest quartile $(P=0.027$ and 0.046 , respectively). Additionally, Table 5 also presents the same indices of Fe status across quartiles of visceral fat mass. In boys, serum Fe and TS differed significantly among quartiles $(P<0.05)$, being lower in the highest than in the lowest quartile. On the other hand, TIBC and ferritin differed significantly among quartiles $(P<0 \cdot 05)$, but were higher in the highest than in the lowest quartile. As far as girls were concerned, TIBC was higher and TS was lower in the highest compared to the lowest quartile of visceral fat mass $(P<0 \cdot 05)$.

The logistic regression analysis showed that overweight boys and obese girls were $2 \cdot 13$ (95\% CI, $1 \cdot 27,3 \cdot 60)$ and $2 \cdot 25$ (95\% CI 1.14, 4.46) times more likely to be Fe deficient than normal-weight boys after controlling for several important covariates (Table 6). Furthermore, centrally obese girls were $2 \cdot 23$ (95\% CI, 1.30, 3.83) times more likely to be Fe deficient in comparison with girls of normal WC. Boys in the highest quartiles of percentage body fat and visceral fat mass were $2 \cdot 48$ (95\% CI, $1 \cdot 26,4 \cdot 88)$ and $2 \cdot 12$ (95\% CI, 1.07, 4.19) times more likely to be Fe deficient than boys in the lowest quartiles (Table 6). Similarly, girls in the highest quartiles of percentage body fat and visceral fat mass were $2 \cdot 12$ (95\% CI, 1.07, 4.20) 


\section{N British Journal of Nutrition}

Table 2. Biochemical and dietary indices of iron status across BMI groups in prepubertal children

(Mean values and standard deviations)

\begin{tabular}{|c|c|c|c|c|c|c|c|c|c|c|c|c|c|c|}
\hline \multirow[b]{3}{*}{ BMI groups } & \multicolumn{7}{|c|}{ Boys } & \multicolumn{7}{|c|}{ Girls } \\
\hline & \multicolumn{2}{|c|}{$\begin{array}{l}\text { Normal-weight } \\
\quad(n 421)\end{array}$} & \multicolumn{2}{|c|}{$\begin{array}{l}\text { Overweight } \\
\quad(n \text { 226) }\end{array}$} & \multicolumn{2}{|c|}{$\begin{array}{l}\text { Obese } \\
(n 93)\end{array}$} & \multirow[b]{2}{*}{$P^{*}$} & \multicolumn{2}{|c|}{$\begin{array}{l}\text { Normal-weight } \\
\quad(n \text { 461) }\end{array}$} & \multicolumn{2}{|c|}{$\begin{array}{l}\text { Overweight } \\
\quad(n \text { 215) }\end{array}$} & \multicolumn{2}{|c|}{$\begin{array}{l}\text { Obese } \\
(n 77)\end{array}$} & \multirow[b]{2}{*}{$P^{*}$} \\
\hline & Mean & SD & Mean & SD & Mean & SD & & Mean & SD & Mean & SD & Mean & SD & \\
\hline \multicolumn{15}{|l|}{ Biochemical serum indices } \\
\hline $\mathrm{Fe}(\mu \mathrm{g} / \mathrm{l})$ & $905 \dagger \ddagger$ & 329 & $836 \dagger$ & 354 & $816 \ddagger$ & 304 & 0.024 & 920 & 349 & 926 & 340 & 835 & 361 & $0 \cdot 166$ \\
\hline TIBC $(\mu \mathrm{g} / \mathrm{l})$ & 3338 & 534 & 3372 & 515 & 3462 & 430 & 0.160 & $3422 \dagger \ddagger$ & 535 & $360 \cdot 2 \dagger$ & 610 & $3600 \ddagger$ & 943 & 0.002 \\
\hline TS (\%) & $27 \cdot 3 \dagger \ddagger$ & 9.5 & $25 \cdot 2 \dagger$ & $10 \cdot 6$ & $23.5 \ddagger$ & $8 \cdot 2$ & 0.002 & $27 \cdot 2 \ddagger$ & $10 \cdot 2$ & $26 \cdot 1$ & $9 \cdot 7$ & $23 \cdot 7 \ddagger$ & $10 \cdot 3$ & 0.032 \\
\hline Ferritin (ng/ml) & $30 \cdot 6 \dagger \ddagger$ & $20 \cdot 7$ & $35 \cdot 3 \dagger$ & $22 \cdot 9$ & $35 \cdot 8 \ddagger$ & $19 \cdot 1$ & 0.021 & $27 \cdot 3 \ddagger$ & $15 \cdot 5$ & $30 \cdot 5$ & $19 \cdot 4$ & $31 \cdot 6 \ddagger$ & 14.4 & 0.036 \\
\hline \multicolumn{15}{|l|}{ Dietary indices } \\
\hline Fe intake $(\mathrm{mg} / 4184 \mathrm{~kJ}(\mathrm{mg} / 1000 \mathrm{kcal}))$ & $5 \cdot 8$ & 1.9 & $6 \cdot 3$ & $2 \cdot 2$ & 5.9 & $2 \cdot 9$ & 0.246 & $6 \cdot 0$ & 4.4 & $6 \cdot 7$ & 3.6 & $6 \cdot 4$ & $3 \cdot 9$ & 0.176 \\
\hline Fe status categories (\% of total) & \multirow{3}{*}{\multicolumn{2}{|c|}{$10.7 \S$ व }} & & & & & & & & & & \multirow{3}{*}{\multicolumn{2}{|c|}{$\begin{array}{l}26 \cdot 6 \rrbracket \\
9 \cdot 49 \text { ** }\end{array}$}} & \\
\hline ID & & & \multicolumn{2}{|c|}{$20.7 \S$} & \multicolumn{2}{|c|}{$16 \cdot 09$} & $0.009 \|$ & \multicolumn{2}{|c|}{12.99} & \multicolumn{2}{|c|}{$13 \cdot 9$} & & & $0.017 \|$ \\
\hline IDA & & & & & & & $0 \cdot 113 \|$ & & & & & & & $0.002 \|$ \\
\hline
\end{tabular}

TIBC, total Fe-binding capacity; TS, transferrin saturation; ID, Fe deficiency with or without anaemia; IDA, Fe-deficiency anaemia

"Derived from ANOVA.

† Mean values were significantly different between normal-weight and overweight children after post-hoc multiple comparisons ( $P<0.05$, Bonferroni rule).

¥ Mean values were significantly different between normal-weight and obese children after post-hoc multiple comparisons ( $P<0.05$, Bonferroni rule).

$\S$ Values were significantly different between normal-weight and overweight children $(P<0.05$, two-sample $Z$-test).

Derived from the Pearson $\chi^{2}$ test.

I Values were significantly different between normal-weight and obese children $(P<0.05$, two-sample $Z$-test)

${ }_{* \star}^{*}$ Values were significantly different between over-weight and obese children $(P<0.05$, two-sample $Z$-test $)$.

Table 3. Biochemical and dietary indices of iron status across waist circumference (WC) groups in prepubertal children

(Mean values and standard deviations)

\begin{tabular}{|c|c|c|c|c|c|c|c|c|c|c|}
\hline \multirow[b]{3}{*}{ WC groups } & \multicolumn{5}{|c|}{ Boys } & \multicolumn{5}{|c|}{ Girls } \\
\hline & \multicolumn{2}{|c|}{ Normal WC (n 613) } & \multicolumn{2}{|c|}{$\begin{array}{c}\text { Central obesity* }(n \\
127)\end{array}$} & \multirow[b]{2}{*}{$P \dagger$} & \multicolumn{2}{|c|}{ Normal WC ( $n$ 614) } & \multicolumn{2}{|c|}{$\begin{array}{c}\text { Central obesity* }(n \\
139)\end{array}$} & \multirow[b]{2}{*}{$P \dagger$} \\
\hline & Mean & SD & Mean & SD & & Mean & SD & Mean & SD & \\
\hline \multicolumn{11}{|l|}{ Biochemical serum indices } \\
\hline $\mathrm{Fe}(\mu \mathrm{g} / \mathrm{l})$ & 892 & 340 & 769 & 299 & $0 \cdot 165$ & 933 & 349 & 820 & 330 & $0 \cdot 267$ \\
\hline TIBC $(\mu \mathrm{g} / \mathrm{l})$ & 3343 & 526 & 3468 & 461 & 0.285 & 3474 & 563 & 3583 & 811 & 0.153 \\
\hline TS (\%) & $27 \cdot 0$ & 9.9 & $22 \cdot 3$ & $8 \cdot 3$ & 0.033 & $27 \cdot 2$ & $10 \cdot 1$ & 23.4 & $9 \cdot 7$ & 0.304 \\
\hline Ferritin (ng/ml) & $32 \cdot 3$ & 21.5 & 34.5 & 19.9 & 0.683 & $28 \cdot 3$ & $16 \cdot 8$ & $30 \cdot 0$ & $16 \cdot 1$ & 0.799 \\
\hline \multicolumn{11}{|l|}{ Dietary indices } \\
\hline $\mathrm{Fe}$ intake $(\mathrm{mg} / 4184 \mathrm{~kJ}(\mathrm{mg} / 1000 \mathrm{kcal}))$ & 5.9 & 3.4 & $6 \cdot 2$ & $3 \cdot 2$ & 0.483 & $6 \cdot 2$ & $4 \cdot 1$ & 6.5 & 4.2 & 0.563 \\
\hline \multicolumn{11}{|l|}{ Fe status categories (\% of total) } \\
\hline ID & \multirow{2}{*}{\multicolumn{2}{|c|}{$\begin{array}{c}13.5 \\
1.0\end{array}$}} & \multirow{2}{*}{\multicolumn{2}{|c|}{$20 \cdot 2$}} & $0.093 \ddagger$ & \multirow{2}{*}{\multicolumn{2}{|c|}{$12 \cdot 4$}} & \multicolumn{2}{|c|}{$25 \cdot 2$} & $0.001 \ddagger$ \\
\hline IDA & & & & & $0.006 \ddagger$ & & $2 \cdot 0$ & \multicolumn{2}{|c|}{5.4} & $0.042 \ddagger$ \\
\hline
\end{tabular}

TIBC, total Fe-binding capacity; TS, transferrin saturation; ID, Fe deficiency with or without anaemia; IDA, Fe-deficiency anaemia.

WC $\geq 90$ th age- and sex-specific percentile.

†Derived from Student's $t$ test.

$\neq$ Derived from Pearson $\chi^{2}$ 
and 2.17 (95\% CI, 1.09, 4.34) times more likely to be Fe deficient than girls in the lowest quartiles.

\section{Discussion}

The present study is among the first to examine the association of several total and regional body fat indices with biochemical indices of $\mathrm{Fe}$ status in a considerably large and representative sample of preadolescents. Overall, the prevalence of ID was remarkably high for obese and centrally obese children and for children in the highest quartiles of percentage body fat and visceral fat mass, ranging from 16 to $27 \%$. These values are similar or higher than those reported for overweight Swiss and Israeli children and adolescents (i.e. 20 and $12 \%$, respectively) ${ }^{(14,34)}$. In these two studies, as well as in the present one, the definition of ID was based on reliable biochemical markers of Fe status, such as serum transferrin receptor concentration, TS and free erythrocyte protoporphyrin concentration. However, the prevalence of ID reported by the present and the two aforementioned studies was considerably higher compared to similar data from the $\mathrm{USA}^{(12)}$, since, according to the $3 \mathrm{rd}$ National Health and Nutrition Examination Survey, the prevalence of ID in obese children and adolescents was 2.4 and $9 \cdot 1 \%$, respectively. The use of serum ferritin as one of the diagnostic criteria for ID could probably provide an explanation for the lower prevalence of ID observed in the American population. Ferritin is an acute-phase protein and its serum levels are plausibly elevated in states of chronic or acute inflammation ${ }^{(35)}$. Considering that excess total and, especially, visceral fat mass represents a chronic inflammatory state, the increased levels of serum ferritin in overweight and obese children could have contributed to an underestimation of the prevalence of ID in this cohort. This hypothesis is strengthened by a more recent study that also used NHANES data to examine the incidence of ID among overweight female adolescents ${ }^{(10)}$. In this study, serum ferritin was excluded from the definition of ID, resulting in a prevalence value of $30.8 \%$.

Consistent with other studies that have reported a positive association between serum ferritin levels and body fat indices, mainly in adults ${ }^{(23,36,37)}$, the present study has found significantly higher serum ferritin in boys in the highest quartiles of percentage body fat and visceral fat mass, as well as in girls in the highest quartile of percentage body fat, compared to the corresponding lowest quartiles (Tables 2-6). By contrast, the values of the other biochemical indices of Fe status were indicative of depleted body $\mathrm{Fe}$ stores for children in the highest quartiles of percentage body fat and visceral fat mass. In particular, boys and girls in the highest quartiles of percentage body fat and visceral fat mass had significantly lower TS than their peers in the lowest quartiles. This was also true for serum $\mathrm{Fe}$ in most cases. In addition, TIBC of girls in the highest quartiles of percentage body fat and visceral fat mass were significantly higher compared to girls in the lowest quartiles. These findings are consistent with recent studies showing that obese children and adults had

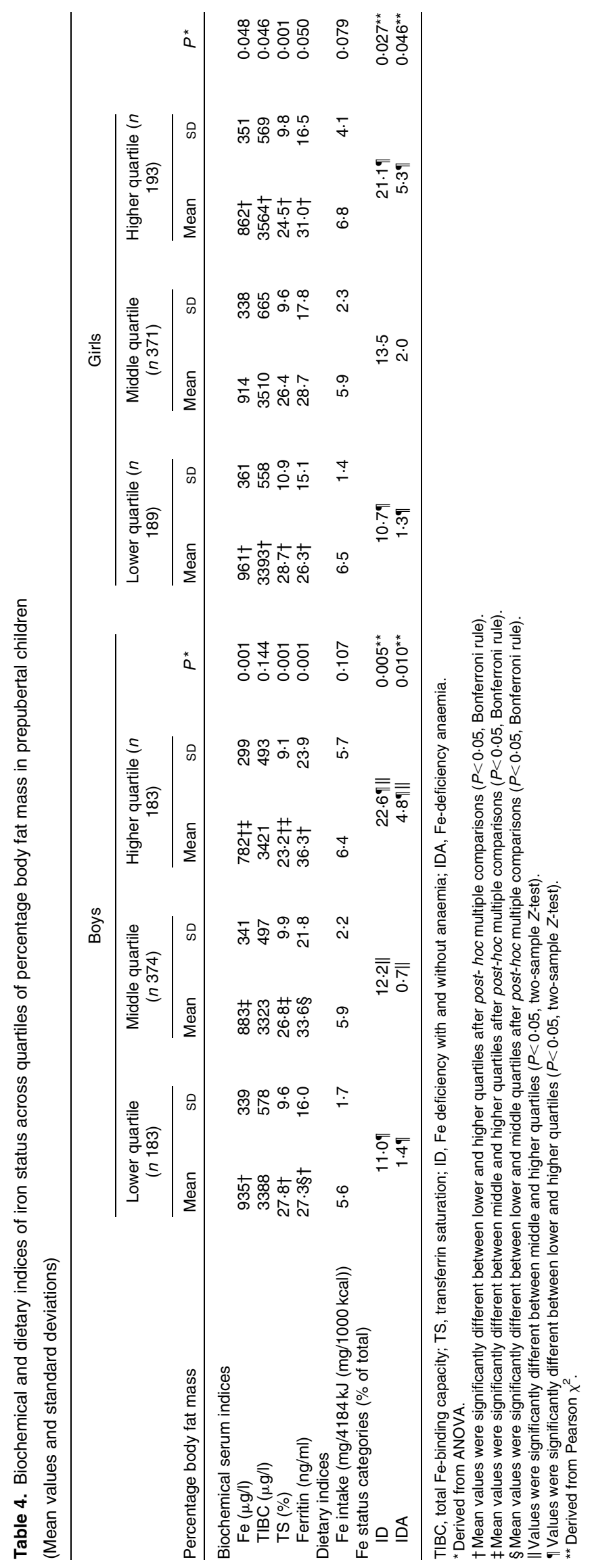


lower levels of serum Fe and TS than their normal-weight counterparts ${ }^{(10,13,16)}$.

The mechanism linking obesity with ID has not yet been elucidated. One proposed such mechanism is low dietary Fe intake by overweight individuals. Still, the available literature in this regard remains controversial. Although a study on Greek adolescents reported low Fe intakes by overweight pupils ${ }^{(38)}$, other recent studies did not find any significant differences in $\mathrm{Fe}$ intake between obese and non-obese children and adolescents ${ }^{(10,16,34)}$. Similarly, the present study did not show any significant differences in dietary Fe intake between the weight and WC groups and among quartiles of total and visceral fat mass (Tables 1-5). Differences in the dietary intakes of specific nutrients that either enhance (vitamin $\mathrm{C}$ ) or inhibit ( $\mathrm{Ca}$, dietary fibre) intestinal nonhaeme $\mathrm{Fe}$ absorption ${ }^{(39)}$ could also provide an explanation for the depleted Fe stores observed in children with increased adiposity. However, Aeberli et al. ${ }^{(34)}$ reported no significant associations between intakes of bioavailable $\mathrm{Fe}$ or factors enhancing Fe absorption and overweight in Swiss children and adolescents.

Furthermore, the bioavailability of Fe may also be modulated by other physiological factors mainly linked to the chronic inflammation induced by excess adiposity. The significant positive associations observed in the present study, between higher percentage body fat and visceral fat mass and ID (Table 6), could strengthen the aforementioned link. More specifically, increased total and visceral fat mass accumulation stimulates the production of inflammatory cytokines $^{(22)}$, that have been reported to be inversely associated with serum Fe levels ${ }^{(10)}$. The hypothesis, that the significant association observed between ID and obesity is mediated by a low-grade chronic inflammation, is also supported by the higher levels of serum ferritin observed among overweight and obese children in the present study. Moreover, serum levels of hepcidin, which is the main regulatory hormone for Fe absorption and recirculation, have been reported by other studies to be significantly higher in overweight children, adolescents ${ }^{(13,34)}$ and adults ${ }^{(10,40)}$ than in normal-weight peers. Adipose-derived cytokines (such as IL-6 and leptin) produced in response to obesity-related inflammation activate hepcidin gene transcription ${ }^{(41)}$. This results in increased serum hepcidin levels that lead to the sequestration of Fe within the reticuloendothelial system and to decreased dietary Fe absorption from the intestine by controlling the expression of ferroportin 1 at the basolateral membranes of the enterocytes ${ }^{(42)}$.

The present study has certain strengths and limitations. Regarding strengths, the Healthy Growth Study is a largescale, epidemiological study covering the central, northern, southern and western parts of the Greek territory. Furthermore, to our knowledge, this is the first study reporting associations between visceral fat mass and Fe status in children. One other strength of the present study relates to the fact that detailed data on diet, blood and anthropometry as well as important confounders (e.g. socio-economic status and pubertal status) were collected. Regarding limitations, additional to its cross-sectional design, the present study has been based on a sub-sample of 1493 pupils with full body 
composition data out of the 2655 pupils with parental consent to participate in the study. Still, no statistically significant differences were observed between pupils with full body composition data (including visceral fat mass levels; i.e. $n$ 1493) and the rest 1162 participating pupils with no visceral fat mass data with regards to parental educational level and weight status (data not shown). Another limitation was the use of only three $24 \mathrm{~h}$ recalls, which may not be sufficient to assess children's habitual dietary intake. Nonetheless, this is of particular concern for foods consumed only occasionally (whereas foods providing Fe are consumed on a more regular basis) while $24 \mathrm{~h}$ recalls are most feasible in larger samples of children such as in the present study.

In conclusion, the present study showed significant positive associations of higher percentage body fat and visceral fat mass with ID in both sexes. These associations may have important public health implications, since they point to the need to also consider excess adiposity as a non-traditional risk factor for ID and IDA. Higher total and visceral fat mass levels should be taken into account when assessing children's body Fe status and should probably be treated before providing dietary recommendations to correct ID or IDA. Future prospective studies could help highlight the basis of the association between obesity and ID, thus providing better insight into how to most effectively tackle the important public health issues of excess adiposity and ID as early in life as possible.

\section{Acknowledgements}

The present research has been co-financed by the European Union (European Social Fund - ESF) and Greek national funds through the Operational Programme Education and Lifelong Learning' of the National Strategic Reference Framework (NSRF) - Research Funding Programme: Heracleitus II. Investing in knowledge society through the European Social Fund. None of the authors has any conflict of interest to declare. All authors contributed to the study design, writing and revising of the manuscript. G. M., V. M. and Y. M. were responsible for the data collection, management and statistical analyses. The authors thank the 'Healthy Growth Study' group for the valuable contribution to the completion of the study.

\section{Healthy Growth Study Group}

Harokopio University Research Team/Department of Nutrition and Dietetics: Yannis Manios (Coordinator), George Moschonis (Project Manager), Katerina P. Skenderi, Evangelia Grammatikaki, Odysseas Androutsos, Sofia Tanagra, Alexandra Koumpitski, Paraskevi-Eirini Siatitsa, Anastasia Vandorou, Aikaterini-Efstathia Kyriakou, Vasiliki Dede, Maria Kantilafti, Aliki-Eleni Farmaki, Aikaterini Siopi, Sofia Micheli, Louiza Damianidi, Panagiota Margiola, Despoina Gakni, Vasiliki Iatridi, Christina Mavrogianni, Kelaidi Michailidou, Aggeliki Giannopoulou, Efstathoula Argyri, Konstantina Maragkopoulou, Maria Spyridonos, Eirini Tsikalaki, Panagiotis Kliasios, Anthi Naoumi, Konstantinos Koutsikas, Katerina Kondaki, Epistimi Aggelou, Zoi Krommyda, Charitini Aga, Manolis 
Birbilis, Ioanna Kosteria, Amalia Zlatintsi, Elpida Voutsadaki, Eleni-Zouboulia Papadopoulou, Zoi Papazi, Maria Papadogiorgakaki, Fanouria Chlouveraki, Maria Lyberi, Nora Karatsikaki-Vlami, Eva Dionysopoulou, Efstratia Daskalou.

Aristotle University of Thessaloniki/School of Physical Education and Sports Sciences: Vassilis Mougios, Anatoli Petridou, Konstantinos Papaioannou, Georgios Tsalis, Ananis Karagkiozidis, Konstantinos Bougioukas, Afroditi Sakellaropoulou, Georgia Skouli.

University of Athens/Medical School: George P. Chrousos, Maria Drakopoulou, Evangelia Charmandari, Neni Pervanidou.

\section{References}

1. Cattaneo A, Monasta L, Stamatakis E, et al. (2010) Overweight and obesity in infants and pre-school children in the European Union: a review of existing data. Obes Rev 11, 389-398.

2. Ogden CL, Carroll MD, Curtin LR, et al. (2009) Prevalence of high body mass index in US children and adolescents, 2007-2008. JAMA 303, 242-249.

3. Wang Y \& Lobstein T (2006) Worldwide trends in childhood overweight and obesity. Int J Pediatr Obes 1, 11-25.

4. Manios Y, Magkos F, Christakis G, et al. (2005) Twenty-year dynamics in adiposity and blood lipids of Greek children: regional differences in Crete persist. Acta Paediatr 94, 859-865.

5. Jean-Claude D (2006) Our children and the metabolic syndrome. Drug Dev Res 67, 602-606.

6. Gade W, Schmit J, Collins M, et al. (2010) Beyond obesity: the diagnosis and pathophysiology of metabolic syndrome. Clin Lab Sci 23, 51-61, quiz 62-55.

7. Kimmons JE, Blanck HM, Tohill BC, et al. (2006) Associations between body mass index and the prevalence of low micronutrient levels among US adults. MedGenmed 8, 59 .

8. Kaidar-Person O, Person B, Szomstein S, et al. (2008) Nutritional deficiencies in morbidly obese patients: a new form of malnutrition? Obes Surg 18, 870-876.

9. Wenzel BJ, Stults HB \& Mayer J (1962) Hypoferraemia in obese adolescents. Lancet ii, 327-328.

10. Tussing-Humphreys LM, Nemeth E, Fantuzzi G, et al. (2010) Elevated systemic hepcidin and iron depletion in obese premenopausal females. Obesity (Silver Spring) 18, 1449-1456.

11. Brotanek JM, Gosz J, Weitzman M, et al. (2007) Iron deficiency in early childhood in the United States: risk factors and racial/ethnic disparities. Pediatrics 120, 568-575.

12. Nead KG, Halterman JS, Kaczorowski JM, et al. (2004) Overweight children and adolescents: a risk group for iron deficiency. Pediatrics 114, 104-108.

13. Amato A, Santoro N, Calabro P, et al. (2010) Effect of body mass index reduction on serum hepcidin levels and iron status in obese children. Int J Obes (Lond) 34, 1772-1774.

14. Pinhas-Hamiel O, Newfield RS, Koren I, et al. (2003) Greater prevalence of iron deficiency in overweight and obese children and adolescents. Int $J$ Obes Relat Metab Disord 27, 416-418.

15. Cepeda-Lopez AC, Aeberli I \& Zimmermann MB (2010) Does obesity increase risk for iron deficiency? A review of the literature and the potential mechanisms. Int J Vitam Nutr Res 80, 263-270.

16. Menzie CM, Yanoff LB, Denkinger BI, et al. (2008) Obesityrelated hypoferremia is not explained by differences in reported intake of heme and nonheme iron or intake of dietary factors that can affect iron absorption. J Am Diet Assoc 108, 145-148.

17. Lecube A, Carrera A, Losada E, et al. (2006) Iron deficiency in obese postmenopausal women. Obesity (Silver Spring) 14, 1724-1730.

18. Pollitt E (2001) The developmental and probabilistic nature of the functional consequences of iron-deficiency anemia in children. J Nutr 131, 669S-675S.

19. Halterman JS, Kaczorowski JM, Aligne CA, et al. (2001) Iron deficiency and cognitive achievement among schoolaged children and adolescents in the United States. Pediatrics 107, 1381-1386.

20. Lozoff B, Jimenez E \& Wolf AW (1991) Long-term developmental outcomes of infants with iron deficiency. $N$ Engl J Med 325, 687-694.

21. Lozoff B, Smith J, Liberzon T, et al. (2004) Longitudinal analysis of cognitive and motor effects of iron deficiency in infancy. Pediatr Res 55, 23A.

22. Subramanian V \& Ferrante AW Jr (2009) Obesity, inflammation, and macrophages. Nestle Nutr Workshop Ser Pediatr Program 63, 151-159, 259-168.

23. Iwasaki T, Nakajima A, Yoneda M, et al. (2005) Serum ferritin is associated with visceral fat area and subcutaneous fat area. Diabetes Care 28, 2486-2491.

24. Taylor RW, Jones IE, Williams SM, et al. (2002) Body fat percentages measured by dual-energy X-ray absorptiometry corresponding to recently recommended body mass index cutoffs for overweight and obesity in children and adolescents aged 3-18 y. Am J Clin Nutr 76, 1416-1421.

25. Cole TJ, Flegal KM, Nicholls D, et al. (2007) Body mass index cut offs to define thinness in children and adolescents: international survey. BMJ 335, 194.

26. Cole TJ, Bellizzi MC, Flegal KM, et al. (2000) Establishing a standard definition for child overweight and obesity worldwide: international survey. BMJ 320, 1240-1243.

27. Fernandez JR, Redden DT, Pietrobelli A, et al. (2004) Waist circumference percentiles in nationally representative samples of African-American, European-American, and Mexican-American children and adolescents. J Pediatr 145, 439-444.

28. Tanner JM (1955) Growth at Adolescence. Oxford: Blackwell Scientific.

29. Ryo M, Maeda K, Onda T, et al. (2005) A new simple method for the measurement of visceral fat accumulation by bioelectrical impedance. Diabetes Care 28, 451-453.

30. Nielsen BM, Dencker M, Ward L, et al. (2007) Prediction of fat-free body mass from bioelectrical impedance among 9- to 11-year-old Swedish children. Diabetes Obes Metab 9 , 521-539.

31. World Health Organization (2001) Iron Deficiency Anaemia: Assessment, Prevention and Control. A Guide for Programme Managers. Geneva: WHO.

32. Mentzer WC Jr (1973) Differentiation of iron deficiency from thalassaemia trait. Lancet $\mathbf{i}, 882$.

33. Trichopoulou A (2004) Composition Tables of Foods and Greek Dishes. Athens: School of Medicine, Department of Hygiene and Epidemiology.

34. Aeberli I, Henschen I, Molinari L, et al. (2009) Stabilization of the prevalence of childhood obesity in Switzerland. Swiss Med Wkly 140, w13046.

35. Wisse BE (2004) The inflammatory syndrome: the role of adipose tissue cytokines in metabolic disorders linked to obesity. J Am Soc Nephrol 15, 2792-2800.

36. Gillum RF (2001) Association of serum ferritin and indices of body fat distribution and obesity in Mexican American 
men - the Third National Health and Nutrition Examination Survey. Int J Obes Relat Metab Disord 25, 639-645.

37. Wrede CE, Buettner R, Bollheimer LC, et al. (2006) Association between serum ferritin and the insulin resistance syndrome in a representative population. Eur J Endocrinol 154, 333-340.

38. Hassapidou M, Fotiadou E, Maglara E, et al. (2006) Energy intake, diet composition, energy expenditure, and body fatness of adolescents in northern Greece. Obesity (Silver Spring) 14, 855-862.

39. Ahmed F, Coyne T, Dobson A, et al. (2008) Iron status among Australian adults: findings of a population based study in Queensland, Australia. Asia Pac J Clin Nutr 17, 40-47.

40. Tussing-Humphreys LM, Nemeth E, Fantuzzi G, et al. (2010) Decreased serum hepcidin and improved functional iron status 6 months after restrictive bariatric surgery. Obesity (Silver Spring) 18, 2010-2016.

41. Verga Falzacappa MV, Vujic Spasic M \& Kessler R (2007) STAT3 mediates hepatic hepcidin expression and its inflammatory stimulation. Blood 109, 353-358.

42. Zafon C, Lecube A \& Simo R (2009) Iron in obesity. An ancient micronutrient for a modern disease. Obes Rev 11, 322-328. 